\title{
CREENCIAS POPULARES Y ENSEÑANZA DE LA BIOLOGÍA
}

\author{
FONTES, M.A. ${ }^{\mathrm{I}}$ y DUARTE, M.C. ${ }^{2}$ \\ 1. Universidade de Trás-os-Montes e Alto Douro, 5000 Vila Real. Portugal. \\ 2. Universidade do Minho, 4700 Braga. Portugal.
}

\begin{abstract}
SUMMARY
The existence of some popular beliefs has been referred to by several studies. A few of these popular beliefs, which seem to be also widespread in Portugal, are related to biological contents. Therefore, it can be expected that many students hold some of these beliefs when they come to the biology classes.

Based on this suspicion, the authors decided to investigate:

- The degree of adhesion shown by students with different biology backgrounds, to some popular beliefs.

- The importance given by some biology textbooks to the popular beliefs.
\end{abstract}

\section{INTRODUCCIÓN}

Una de las más importantes líneas de investigación de la última década está ligada a la problemática de las iđeas alternativas que poseen alumnos de diferentes grados de escolarización acerca de diversos tópicos científicos. A pesar de que la mayoría de estos trabajos han incidido sobre temas de física, es ya considerable el número de estudios dedicados a diversos tópicos biológicos: fotosíntesis, respiración, evolución, herencia, célula, etc. El análisis de muchos de estos trabajos ha contribuido a una mejor comprensión de la naturaleza y diversidad de las ideas de los alumnos, en cuyo origen se presume que juega un papel importante el medioen que se desenvuelven. Norman (citado por Hewson 1985) hace notar que la manera en que se percibe por parte de las personas el mundo físico es afectada e incluso alterada por su conocimiento cultural.

Aunque se esté en una fase esencialmente especulativa en lo referente al origen đe las ideas alternativas, algunos estudios exploratorios han demostrado la influencia de algunas metáforas culturales en la formación de esas ideas (Hewson 1985); Deadman y Kelly (1978) destacan la utilización de creencias basadas en una combinación de folclore y empirismo para explicar algunos problemas relacionados con ta herencia; folclore, explicitan estos autores, en el sentido que utílizan aspectos del conocimiento popular no relacionados con los principios biológicos.

El ambiente cultural en que viven las personas parece asumir un papel fundamental en las ideas que poseen, y afecta a todo aquello relacionado con su medio externo, incluyendo el aprendizaje escolar.

Son muchos los autores, y no todos ellos actuales, que han recogido referencias sobre la existencia y divulgación de las creencias populares. Ya en el siglo XVH, el padre Jean Thiers (Izard y Smith 1979) decía, refiriéndose a las creencias populares, que existían en todas las clases sociales, desde la grande y pequeña burguesía hasta las personas sencillas, pasando por el pueblo no alfabetizado.

Malinowski (1984) considera que, a pesar de que las creencias no tienen un fundamento científico, pueden ser adoptadas por el hombre moderno que se apoya en la ciencia y en la razón. Para este autor, las fases fisiológicas de la vida del hombre, como la concepción y la gravidez, constituyen el núcleo de muchas de estas creencias. 
En Portugal, una serie de estudios ligados a la etnografía y la antropología han divulgado la existencia de diferentes creencias populares, muchas de las cuales se relacionan con asuntos de biología. Creencias como: «si una mujer grávida huele flores el bebé nace con manchas en la piel», "si se guardan alubias en sacos de plástico, crían bichos», parecen estar ampliamente difundidas entre la población portuguesa, aunque puede haber ligeras variaciones entre unas regiones y otras.

Teófilo Braga (1986) ha descrito de manera detallada muchas de las creencias que permanecen en nuestro país, y Teresa Joaquim (1983), en un ensayo sobre prácticas de gravidez, parto y postparto en Portugal, describe e interpreta muchas creencias, intentando dar un explicación a su origen.

Dado que muchos de los jóvenes que entran en nuestro sistema educativo han vivido y viven inmersos en un medio social donde muchas de estas creencias son predominantes, en las escuelas siguen adoptando algunas de ellas. Teniendo en cuenta este supuesto, y la problemática de las ideas alternativas y su importancia en el proceso de enseñanza-aprendizaje, el objetivo del presente estudio es proporcionar una respuesta a las siguientes cuestiones:

- ¿Hasta qué punto Ios alumnos de diferentes grados de enseñanza presentan creencias populares ligadas a asuntos de biología?

- ¿Hasta qué punto la escuela, particularmente en la enseñanza de la biología, contribuye a desmitificar muchas de estas creencias populares?

\section{METODOLOGÍA}

Muestra. La muestra de este trabajo ha sido de 115 alumnos pertenecientes a la enseñanza secundaria y superior:

-25 alumnos de $10^{\circ}$ año del área científico-natural.

-25 alumnos de $12^{\circ}$ año del área científico-natural.

-30 alumnos de Ir año de la licenciatura en enseñanza de biología/geología.

-35 alumnos de $4^{\circ}$ año de la licenciatura en enseñanza de biología/geología.

Únicamente los alumnos de $10^{\circ}$ no han cursado los contenidos relacionados con las creencias estudiadas.

\section{Instrumento}

Se utilizó un cuestionario, elaborado por las autoras de este trabajo, que fue aplicado a todos los alumnos de la muestra. Const 6 de una lista de 25 creencias populares (ver anexo). En cada una de ellas se pedía al encuestado que señalase con una $X$ la opción correspondiente a su situación u opinión:

-Nunca lo había oído.

-Lo había ó́do, pero no estoy de acuerdo.

-Lo había oído y estoy de acuerdo.

-Lo había oído y estoy indeciso.

De manera paralela se construyó por parte de las autoras una ficha de análisis de libros de texto, en la que se pretendía investigar si en éstos se hacía alguna referencia a la existencia de creencias populares y, en el caso de ser así, cómo relacionaban este conocimiento popular con el conocimiento científico.

\section{DESCRIPCIÓN DEL ESTUDIO}

El cuestionario que fue aplicado a los 115 alumnos de la muestra se elaboró teniendo como base una colección de creencias, recogidas en diferentes puntos del país, relacionadas con contenidos del conocimiento científico del área de biología. Esta colección se llevó a cabo por unos 80 alumnos de $4^{2}$ año de la licenciatura de enseñanza de biología/geología; en el ámbito de la disciplina Didáctica de la Biología; alumnos que no fueron incluidos en la muestra experimental.

De todas las creencias recogidas, fueron seleccionadas 25 de acuerdo con los siguientes criterios:

-Su mayor incidencia en las diferentes zonas de recogida.

-Su grado de reiación con contenidos incluidos en los programas de biología de la enseñanza secundaria.

El segundo de estos criterios permitió distribuir las creencias seleccionadas en tres grupos relacionados con los contenidos: origen de la vida, ciclo reproductor de la mujer y genética.

La aplicación del cuestionario atendió a los siguientes objetivos:

- Comprobar el grado de adhesión y divulgación de las creencias consideradas en alumnos que han recibido diferentes grados de enseñanza de biología.

-Inferir en qué medida la enseñanza de tópicos programáticos de biología, como el origen de la vida, el ciclo reproductor de la mujer y la genética, contribuye a desmitificar algunas de las creencias populares de una determinada parte de la población portuguesa.

El grado de divulgación relativo a cada creencia, se obtuvo a partir de la frecuencia de la respuesta «lo había oído,...», independientemente de que los alumnos estuvieran de acuerdo o no con las creencias, utilizando el siguiente criterio: 
Respuesta: «Lo había oído,...» $\leq 50 \%$ de la muestra grado bajo de divulgacion.

Respuesta: "Lo había oido,..." > 50\% y $\leq 75 \%$ de la muestra grado medio de divulgación.

Respuesta: «Lo había oído,...»> 75\% de la muestra grado alto de divuIgación.

El grado de adhesión relativo a cada creencia, fue inferido por la frecuencia de respuesta «Lo había oído, y estoy de acuerdo».

En lo relativo a la seiección de libros de texto, fueron elegidos tres de $11^{\circ}$ año y dos de $12^{\circ}$ año, por considerarlos los más frecuentes en la zona norte de Portugal. No obstante, después de haber reahizado el análisis, no se encontró en ninguno de los textos referencia alguna al conocimiento popular.

\section{RESULTADOS}

\section{Grado de divulgación}

En la tabla I se presenta el grado de divulgación de las creencias del cuestionario, según el criterio explicitado anteriormente.

Tabla I

Grado de divulgación de las creencias consideradas.

\begin{tabular}{|ll|}
\hline Grado de divulgacion & Creencias \\
\hline Bajo & $\begin{array}{l}4,5,7,8,9,10,11,12,14,15,18,19, \\
20,21,22,23,24 .\end{array}$ \\
\hline Medio & $1,2,13,16,17,25$. \\
\hline Alto & $3,6$. \\
\hline
\end{tabular}

Los resultados permiten observar que de las 25 creencias consideradas, el $68 \%$ son poco conocidas, el $24 \%$ mantienen un grado de divulgación apreciable y apenas el $8 \%$ son muy conocidas, naturalmente dentro de la muestra. Hay que hacer notar que dentro de las creencias más divulgadas, prevalecen las relacionadas con el ciclo reproductor de la mujer $(50 \%)$.

\section{Grado de adhesión}

El grado de adhesión relativo a las creencias consideradas fue únicamente determinado para aquéllas con un grado de divulgación medio o alto. Los resultados obtenidos se recogen en la tabla Il.

La creencia que recibió mayor adhesión en todos los niveles de enseñanza fue: «si se guardan alubias en sacos de plástico, crían bichos", creencia relacionada con la noción de generación espontánea.
Tabla II

Grado de adhesión, en porcentaje, que presentan los alumnos de la muestra a las creencias más conocidas.

\begin{tabular}{|c|c|c|c|c|c|c|c|}
\hline \multirow{2}{*}{$\begin{array}{l}\text { Creencias } \\
\text { Año escolar }\end{array}$} & \multicolumn{2}{|c|}{ Origen de ia vida } & \multirow{2}{*}{$\frac{\text { Genética }}{3}$} & \multicolumn{4}{|c|}{ Ciclo reproductor de la mujer } \\
\hline & 1 & 13 & & 2 & 16 & 17 & 25 \\
\hline $\begin{array}{c}10^{2} \\
(\mathrm{~N}=25)\end{array}$ & 36,0 & 16,0 & 20,0 & & 8,0 & 20,0 & 25,0 \\
\hline $\begin{array}{c}12^{2} \\
(\mathrm{~N}=25)\end{array}$ & 24,0 & & 4,0 & 16,0 & & 12,0 & \\
\hline $\begin{array}{c}\downarrow^{s} \\
(N=30)\end{array}$ & 37,0 & 3,3 & & & & & 14,2 \\
\hline $\begin{array}{c}4^{9} \\
(N=35)\end{array}$ & 26,0 & & & & & 2,8 & \\
\hline
\end{tabular}

El subgrupo de alumnos de $10^{\circ}$ año de escolaridad es el que muestra un mayor grado de adhesión global a las creencias del cuestionario, hecho que podemos considerar normal si pensamos que los niños de este año de escolaridad aún no han recibido una enseñanza formal relativa a la mayoría de los contenidos relacionados con las creencias consideradas, exceptuandoun posible tratamiento muy elemental sobre el origen de la vida en el año preparatorio. De los resultados obtenidos queremos hacer notar:

-Un porcentaje relativamente elevado de alumnos de $12^{\circ}$ año (16\%) y de $1 \mathrm{r}$ año de licenciatura (14\%) muestran adhesión, respectivamente, a las creencias: «si una mujer menstruada bate claras, no se montan» y «si una mujer menstruada hace mahonesa se corta», relacionadas con el ciclo reproductor de la mujer, a pesar de haberlo ya contemplado en la enseñanza reglada en años anteriores.

-El alto porcentaje de alumnos, de todos los años de escolaridad estudiados, manifiestan estar de acuerdo con: «si se guardan alubias en sacos de plástico, crían bichos». Merece especial referencia el hecho de que los alumnos de $4^{2}$ año de licenciatura en enseñanza de bología/geología llegaran prácticamente a fin de curso sin que esta creencia hubiese sido superada.

\section{Grado de indecisión}

Merecen tratamiento especial las respuestas de los alumnos que inđicaban indecisión respecto a su adhesión a las creencias consideradas, por parecernos que esta actitud puede ser un indicador de que una creencia no ha sido totalmente superada en la escolarización.

La tabla III representa el porcentaje de alumnos que manifiestan indecisión frente a las creencias consideradas.

La apreciación global de los resultados permite comprobar que en siete de las ocho creencias consideradas existe un grado no đespreciable de indecisión en prácticamente todos los años de escolaridad estudiados. Es nuevamente la creencia «si se guardan alubias en sacos 
Tabla III

Grado de indecisión, en porcentaje, que presentan los alumnos de la muestra a las creencias más conocidas.

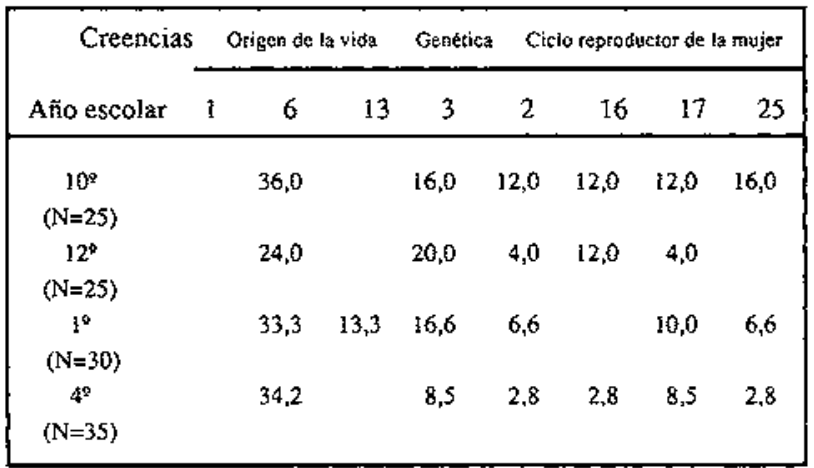

de plástico, crían bichos», la que registra los mayores porcentajes.

Merece destacarse el hecho de que en seis de las ocho creencias consideradas, hay alumnos de $4^{\circ}$ año de licenciatura que se sienten indecisos a la hora de juzgar la veracidad de estas creencias populares.

\section{CONCLUSIONES}

Los resultados presentados parecen permitir extraer algunas conclusiones, todavía con alguna cautela dado el tamaño reducido de la muestra y las limitaciones inherentes al formato estructurado del cuestionario utilizado:

1. Algunas de las creencias inciuidas en el cuestionario presentan un grado de adhesión considerable entre alumnos bastante escolarizados, lo cual no es de extrañar si se tiene en cuenta que la socialización primaria de estos

\section{NOTA}

Este artículo ha sido traducido del original portugués. alumnos se ha realizado en un ambiente donde muchas de estas creencias son tomadas como conocimiento verdadero.

2. La enseñanza de la biología, en lo concerniente a los tópicos relacionados con las creencias consideradas, parece no haber conseguido alterar el «conocimiento popular» con que muchos alumnos liegan a las aulas; esto se verifica con el porcentaje considerable de alumnos que en breve serán habilitados como profesores de biología, y que dudan o se adhieren a algunas de estas creencias populares.

\section{IMPLICACIONES PARA LA ENSEÑANZA}

Tomando en cuenta los resultados y las conclusiones obtenidas en este trabajo, nos parece útil señalar:

1. Es fundamental que el profesor de biología reconozca la importancia del «conocimiento popular» en la construcción del conocimiento científico por parte de los alumnos.

2. El reconocimiento de esta importancia pasa por el diagnóstico y valoración de creencias relacionadas con tópicos de la biología, en el proceso de enseñanza/ aprendizaje para que puedan ser desmitificadas a la luz del conocimiento científico actual.

3. Debería contemplarse en los Iibros de texto escolares los tópicos programáticos que se relacionen con estas creencias, hacer referencia concreta a ellas y apuntar estrategias para su superación.

4. Los formadores responsables de la formación inicial de los futuros profesores de biología, deben ser conscientes de la importancia que esta problemática presenta, y valorarla apropiadamente en sus aulas.

\section{REFERENCIAS BIBLIOGRÁFICAS}

BRAGA, T., I986. O povo português nos seus costumes. Crenças e tradições. (Dom Quixote: Lisboa).

DEADMAN, J.A. y KELLY, P., 1978. What secondary school boys understand about evolution and heredity before they are taught the topics?, Journal of Biological Education, I, pp. $7-15$.

HEWSON, M., 1985. The role of intelectual environment in the origin of conceptions: an exploratory study, en West y Pine, (eds.) Cognitive structure and conceptual change, pp. 153161. (Academic Press).
IZARD, M. y SMITH, P., 1979. La fonction simbolique. (GaIlimard: París).

JOAQUIM, T., 1983. Dar à luz. Ensaio sobre as prácticas $e$ crenças de gravidez, parto e pos-parto, em Portugal. (Dom Quixote: Lisboa).

MALINOWSKI, B., 1984. Magia, Ciência e Religiāo. (Ed. 70: Lisboa). 
ANEXO:

\section{LISTA DE LAS CREENCIAS CONTENIDAS EN EL CUESTIONARIO}

1. Comer castañas crudas hace nacer piojos.

2. Si una mujer menstruada bate claras, no se montan.

3. Si una mujer grávida huele flores, el bebé nace con manchas en la piel.

4. Si una mujer menstruada sube a un árbol, éste se seca.

5. Si una mujer grávida se pone hílos en el cuello, el bebé nace con el cordón umbilical enrollado al cuello.

6. Si se guardan alubias en sacos de plástico, crían bichos.

7. Si se mata un cerdo en Iuna nueva, su carne cría bichos.

8. Si un cerdo se alimenta con desperdicios, el jamón cría bichos.

9. Si una mujer menstruada mata un cerdo, la sangre no sirve para los embutidos.

10. Si una mujer grávida pega a un animal con pelo, el bebé nace con manchas peludas.

11. Si una mujer grávida lleva las llaves en su bolso, el bebé nace con el labio abierto.

12. Si una mujer grávida se pone una liave en la boca, el bebé nace con el labio abierto.

13. Contar estrellas hace salir verrugas.
14. Si una mujer grávida come pulpo, el bebé nace con una especie de ventosas en la piet.

15. Si una mujer grávida usa bragas estampadas con flores, el bebé nace con señales iguales a las flores.

16. Si una mujer menstruada va al cementerio, entran en ella los espíritus.

17. Si una mujer menstruada hace pasteles, no sube la masa.

18. Si una mujer grávida desmenuza bazo o hígado de animales, el bebé nace con manchas rojas.

19. Si una majer grávida limpia pescado, el bebé nace con escamas en la piel.

20. La came de cerdo ahumada en viernes cría bichos.

21. Si una mujer grávida lleva una tijera en el bolso, el bebé nace con el labio partido.

22. Si una mujer grávida usa medalias, el bebé nace con una mancha parecida a la medalla.

23. Si una mujer grávida pasa por debajo de una cuerda, el bebé nace con el cordón umbilical enrollado al cuello.

24. Si una mujer menstruada saca vino de un tonel, éste se echa a perder.

25. Si una mujer menstruada hace mahonesa se corta. 